Annales Geophysicae (2003) 21: 2059-2072 (C) European Geosciences Union 2003

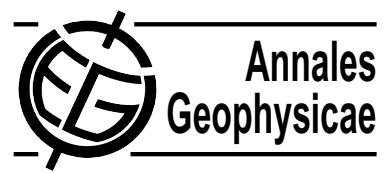

\title{
The occurrence frequency of upward ion beams in the auroral zone as a function of altitude using Polar/TIMAS and DE-1/EICS data
}

\author{
P. Janhunen ${ }^{1}$, A. Olsson ${ }^{2}$, and W. K. Peterson ${ }^{3}$ \\ ${ }^{1}$ Finnish Meteorological Institute, Geophysical Research, Helsinki, Finland \\ ${ }^{2}$ Swedish Insititute of Space Physics, Uppsala Division, Uppsala, Sweden \\ ${ }^{3}$ LASP, University of Colorado, Boulder, Colorado, USA
}

Received: 12 September 2002 - Revised: 16 January 2003 - Accepted: 6 February 2003

\begin{abstract}
We study the occurrence frequency of upward auroral ion beams as a function of altitude using three years of Polar/TIMAS ion data combined with 11 years of DE1/EICS ion data, in order to reach a complete altitude coverage between 5000 and $30000 \mathrm{~km}$. The most interesting result is that there is a peak in ion beam occurrence frequency and invariant energy flux and invariant particle flux at $\sim 3 R_{E}$ radial distance. The peak exists at about the same altitude in both the evening and midnight MLT sectors. No solar cycle effects are found. We suggest that the peak could be due to a preferred altitude of auroral potential structures at $\sim 3 R_{E}$. To substantiate the suggestion, we also present a simple Monte Carlo simulation of ion beams. Another result is that the ion beam occurrence frequency and invariant (mapped to ionospheric altitude) energy and particle fluxes increase in the radial distance range $4-6 R_{E}$, suggesting that wave heating processes may take place in this altitude range.
\end{abstract}

Key words. Magnetospheric physics (auroral phenomena; magnetosphere-ionosphere interactions) - Space plasma physics (charged particle motion and acceleration)

\section{Introduction}

Discrete auroral arcs are associated with inverted-V type accelerated electron precipitation (Frank and Ackerson, 1971). The electron acceleration takes place in an upward parallel electric field residing at $\sim 3000-11000 \mathrm{~km}$ altitude range (the altitude depends on season). The parallel electric field belongs to the bottom part of a U-shaped potential structure. Besides accelerating electrons downward, the parallel electric field also accelerates ionospheric ions upward, forming upgoing ion beams above the bottom of the potential structure (McFadden et al., 1998). The mean energy of the beam ions is expected to agree at least approximately with the magnitude of the potential drop, although care in interpretation is needed because different species are energized differently

Correspondence to: A. Olsson (ao@irfu.se)
(Möbius et al., 1998). Other closely related auroral phenomena are ion conics (Kondo et al., 1990), but they are related to perpendicular wave heating and thus do not give information about the potential structure (André and Yau, 1997). Indications of a combination of parallel acceleration and perpendicular heating (called bimodal acceleration by Klumpar et al., 1984) have also been found in the form of conics elevated in energy (Miyake et al., 1996). Although this much is known about discrete auroral arc formation at low altitude, important questions remain open, such as what is the morphology of the potential structure at higher altitude and what is the physical energy transfer mechanism maintaining the whole potential structure.

Studying the altitude dependence of ion beams is thus one way to approach the physical question of what causes and maintains the inverted-V machinery of auroral electron acceleration, and one which has not been widely pursued yet. However, some studies on the topic have been done. The altitude dependence of ion beams was studied statistically by Yau et al. (1984) and Kondo et al. (1990), both using DE-1 data (8000-24 $000 \mathrm{~km}$ altitude range). The Yau et al. (1984) study uses 1981-82 data while Kondo et al. (1990) use 198186. For high $K_{p}$ values, a minimum in ion beam occurrence frequency for both oxygen and hydrogen was found at around $16000 \mathrm{~km}$ altitude (except for energies less than $1 \mathrm{keV}$ for which the occurrence frequency was monotonically increasing as a function of altitude). Studying this phenomenon further is one of the motivations for the present study.

In order to obtain the maximum information that ion beam altitude dependence can give about auroral acceleration mechanisms, one has to study the altitude dependence also as a function of magnetic local time (MLT) and invariant latitude (ILAT), as well as for different $K_{p}$, solar illumination and solar cycle phase conditions. We now briefly review previous results for ion beam dependence on these parameters and put them into the context of other auroral processes. Ion beam occurrence frequencies as a function of MLT and integrated over altitude appeared in Gorney et al. (1981), Fig. 3, and Yau et al. (1985), Fig. 8. The maximum 
number of fluxes in $\mathrm{O}^{+}$ion beams were found to occur in the pre-midnight sector, which is probably due to the fact that inverted-V phenomena are the most common there (Lin and Hoffman, 1979). Statistical plots of the ILAT dependence of the occurrence frequency (integrated over altitude) appeared in Yau et al. (1985). The low energy ion beam ILAT versus MLT dependence closely followed the ILAT versus MLT dependence of the auroral oval, which is another indication that ion beams are closely associated with inverted- $\mathrm{V}$ electron acceleration. Regarding $K_{p}$ dependence (Fig. 5 of Yau et al. (1985) and Fig. 8 of Kondo et al. (1990)), it was found that the ion fluxes increase as a function of $K_{p}$, which probably reflects the general increase in auroral processes with $K_{p}$. Using one year of TIMAS data it has also been found that ion beams below $10000 \mathrm{~km}$ occur $\sim 3$ times more often during wintertime than during summertime (Collin et al., 1998). This probably reflects the fact that during summertime, potential structures and their associated ion beams often reside at higher altitude, because of higher underlying ionospheric plasma density and thus were more often beyond the upper altitude limit of this study. What is missing in these studies is an altitude dependence together with the other previous parameters. This is the subject of the present paper.

In this paper we study the ion beams statistically as a function of altitude using two different ion instruments, TIMAS and EICS, from two satellites, Polar and DE-1, respectively. The main reason for selecting Polar is the fact that it covers all altitudes above $5000 \mathrm{~km}$, up to and exceeding $30000 \mathrm{~km}$ which we set as the upper limit of our study. The TIMAS instrument on board Polar suffered a high-voltage breakdown on 8 December 1998 and thus the altitude coverage is incomplete. We use DE-1/EICS to complete the altitude coverage. DE-1/EICS is similar to TIMAS, except that the time and energy resolutions are somewhat inferior. The main new topics that we will study are the statistical altitude profile of ion beam occurrence frequency up to $30000 \mathrm{~km}$, and how the altitude profile of ion beams depends on MLT, ILAT, etc. This paper is part of a larger ongoing effort where we look at the altitude dependence of several inverted- $\mathrm{V}$ related phenomena, such as density cavities, potential structures, broad-band electrostatic waves and electron anisotropies. The overarching goal is to study the physical mechanisms of electron acceleration in discrete auroral arcs. For this purpose one needs information about the plasma physical processes in a wide altitude range in auroral flux tubes.

Investigation of counterstreaming ion beams lead Sagawa et al. (1987) and Horita et al. (1987) to infer the existence of a downward directed electric field at high altitudes. More specifically, Janhunen et al. (1999) and Janhunen and Olsson (2000) proposed that the low-altitude U-shaped potential contours often close below 4-5 $R_{E}$ radial distance, forming an "O-shaped" potential. Support for the idea has later come from simulations (Janhunen and Olsson, 2002; Janhunen et al., 2003), Polar/FAST conjunctions (Janhunen et al., 2001), and studies of hemispherical conjugacy of auroras (Hallinan and Stenbaek-Nielsen, 2001). The effect of a closed negative potential structure on ion beams is that the beam which is energized at the bottom of the structure should become decelerated at the top of the structure. In a statistical study, time histories of individual ion beams surely cannot be followed, but the phenomenon should be seen as a reduction of the ion beam occurrence frequency at the closure altitude of the potential contours. Testing the closed potential structure hypothesis from the ion beam point of view is one of the motivations of the paper.

\section{Instrumentation and data analysis}

We use Polar/TIMAS for covering the altitude ranges 500010000 and 20000-32000 km during 1996-1998 (Shelley et al., 1995). TIMAS suffered a high-voltage breakdown in 8 December 1998 and experienced a loss of telemetry for portions of 1999, 2000 and 2001. Although TIMAS reinitiated routine operations on 30 March 2001, the high-voltage breakdown of 1998 resulted in a loss of sensitivity that makes intercomparisons of statistical databases obtained before and after 8 December 1998 too challenging. We chose not to use TIMAS data acquired after 8 December 1998 in this analysis. This makes the altitude coverage of TIMAS incomplete: The EICS instrument on board DE-1 made measurements during 1981-1991, covering altitudes between 8000-23000 km (Shelley et al., 1981). Using both instruments together we can thus obtain a complete altitude coverage from $5000 \mathrm{~km}$ to $30000 \mathrm{~km}$.

In order to be able to use Polar/TIMAS and DE-1/EICS in the same statistics, careful intercalibration is necessary. This will be addressed in Sect. 2.4 ("Polar/TIMAS and DE1/EICS intercomparison").

\subsection{Data sets}

The Polar/TIMAS instrument produces the differential energy flux for all pitch angles and energies with $15^{\circ}$ pitch angle bins and 28 logarithmically spaced energy steps between $15 \mathrm{eV}$ and $33 \mathrm{keV}$ every two satellite spins (about $12 \mathrm{~s}$ ). Before June 1996 the upper energy limit was $25 \mathrm{keV}$, however. The data files used in this study are version 2 high resolution files from the online database of instrument data from many satellites provided by NASA on the CDAWeb site. The DE$1 /$ EICS instrument is very similar to Polar/TIMAS. However, the time resolution of the CDAWeb data files we are using is 16 spins ( $96 \mathrm{~s}$, i.e. an 8 times lower time resolution than for Polar/TIMAS) and also the energy range is more restricted than TIMAS ( $10 \mathrm{eV}$ to $17 \mathrm{keV})$. Our method for finding ion beams requires at least 2 energy channels above the peak energy; thus, the maximum peak energy with DE-1/EICS is $10 \mathrm{keV}$. In order to compare the data sets with the same resolution and restrictions, the energy range of TIMAS is adjusted to the capabilities of EICS, and each EICS data point is replicated 8 times to match Polar/TIMAS time resolution.

The data sets we are using are the best that exist from these instruments. The only compromise in the data quality 
in these sets is that gyrotropy has been assumed throughout, but this is no problem in the present study.

After this paper was written, an error was noticed in Polar/TIMAS telemetry processing which may affect the dayside flux values in the 10-14 MLT range. Consequently, the flux values in this MLT range appearing in Figs. 8 and 9 below may be slightly in error. No conclusions are drawn in this paper concerning this MLT range.

\subsection{Finding ion beams}

We identify the ion beams as energy-angle structures by the following method based on Gaussian fitting. Ion beams are usually defined as maxima in phase space density, but we choose to define them as peaks in the differential energy flux. Let $F(E, \theta)$ denote the measured differential energy flux, where $E$ is the energy and $\theta$ is the pitch angle. Upward ion beams are maxima of $F(E, \theta)$ appearing close to $\theta=\pi$ for the Northern Hemisphere (for the Southern Hemisphere they appear close to $\theta=0$ ). Particulary at higher altitude they are often superposed with a smooth background which is almost symmetric in the up/down direction. For each energy step, to separate the background we first subtract the downgoing part from the upgoing part and replace possible negative values by zero after subtraction. We then find all local maxima along $\theta=\pi$. We consider only maxima that exceed the background by at least $50 \%$. After locating the maximum, the 2-D region in energy and pitch angle around the maximum which belongs to the same ion beam is found. The criteria used here are such that only values that exceed the local background flux by at least $30 \%$ are eligible, and the values must be decreasing as one moves away from the maximum. We also require that the differential energy flux is at least $10^{5} \mathrm{~cm}^{-2} \mathrm{~s}^{-1} \mathrm{sr}^{-1}$. A Gaussian function in energy and pitch angle is then fitted to the maximum region. If the chi-squared of the fit is larger than 1 , the fit is rejected. The energy and number fluxes belonging to the maximum region are then computed, scaled by particle flux conservation in a magnetic flux tube to ionospheric altitude and then saved. In the scaling to ionospheric altitude the value of the magnetic field is needed; this is taken from the Polar/MFE, if available, otherwise, the dipole model is used. In the rare event of having several eligible local maxima in energy, we select the one with the largest number flux. The processing of TIMAS and EICS data is identical except for the different energy grids.

We use several criteria to protect ourselves against different types of data errors. Passages of data with known instrument errors are removed. A data point is excluded if the integrated invariant energy flux ("invariant" means that the quantity is scaled to ionospheric altitude) exceeds $10^{4} \mathrm{~mW} \mathrm{~m}^{-2}$ or if the invariant number flux exceeds $10^{14} \mathrm{~m}^{-2} \mathrm{~s}^{-1}$. These values are too high to be realistic (physical energy fluxes values may reach a few hundred $\mathrm{mW} \mathrm{m}^{-2}$ at most) and must correspond to erroneous flux values. Between 1 April 1996 and 8 December 1998, Polar/TIMAS had 23.9 days worth of valid nightside auroral crossing data (ILAT $=65 \ldots 74$ ) and 27 days worth of missing data. The number of bad data points was only 2 (corresponding to total time $24 \mathrm{~s}$ ). For DE$1 / \mathrm{EICS}$, the percentage of bad data points is $0.9 \%$ and the total amount of nightside auroral zone time is 59 days. By bad data, here, we mean times when the instrument was on and produced data, but the numbers produced do not make physical sense (e.g. were identically zero).

We limit ourselves only to invariant energy fluxes above $0.2 \mathrm{~mW} \mathrm{~m}^{-2}$ and to ion beam energies in the range from $0.5 \mathrm{keV}$ to $10 \mathrm{keV}$. There are plenty of ion beams also below $0.5 \mathrm{keV}$ but we shall not include them in this study because we are primarily interested in those inverted- $\mathrm{V}$ phenomena that relate to visible auroral arcs whose accelerating potentials are of the order of $0.5-1 \mathrm{keV}$ or more. As mentioned above, by invariant energy flux and invariant particle flux we mean that the quantities are projected to the ionospheric level. No distinction between ion species is made. A further condition that we use is that the integrated ion energy flux must be upward at the time of the ion beam, otherwise, the beam is rejected.

\subsection{Computation of occurrence frequencies}

We want to compute the ion beam occurrence frequency as a function of different variables, among which are the radial distance $R$, the invariant latitude (ILAT), the magnetic local time (MLT), the $K_{p}$ index value and solar illumination condition (i.e. whether the ionospheric footpoint is illuminated or not). We do not have enough orbital coverage to obtain the occurrence frequency in the whole five-dimensional array; however, in any event, it would not be practical to plot. Instead, we typically display the dependence of the occurrence frequency on one of the important parameters, as a function of radial distance and averaged over all ILAT. There are, in principle, two ways to gather the statistics: (1) one puts all data points in the radial bin together and computes the occurrence frequency for them, (2) one calculates the occurrence frequency separately for each ILAT bin and computes the arithmetic average of the occurrence frequencies. Method (1) yields longer data vectors and thus smaller statistical uncertainties, but is vulnerable to possibly nonuniform orbital coverage in ILAT. Method (2) does not require uniform ILAT coverage, but gives larger statistical uncertainties if at least one of the ILAT bins contains a significantly smaller number of data points than the other bins. If $f_{i}$ is the occurrence frequency in the $i$ th ILAT bin and $\sigma_{i}^{2}$ is the corresponding variance, the standard deviation of the whole radial bin is given by $(1 / n) \sqrt{\sum_{i} \sigma_{i}^{2}}$, where $n$ is the number of ILAT bins ( 9 in our case).

We chose to use method (2) in this paper, with the additional assumption that if one of the ILAT bins has less than 100 measurements, it is dropped from the statistics, so that it does not contribute to the total standard deviation.

\subsection{Polar/TIMAS and DE-1/EICS intercomparison}

The top panel of Fig. 1 shows all ion beams between 0.5 and $10 \mathrm{keV}$ as a function of radial distance $R$ and beam peak 


\section{Polar TIMAS}
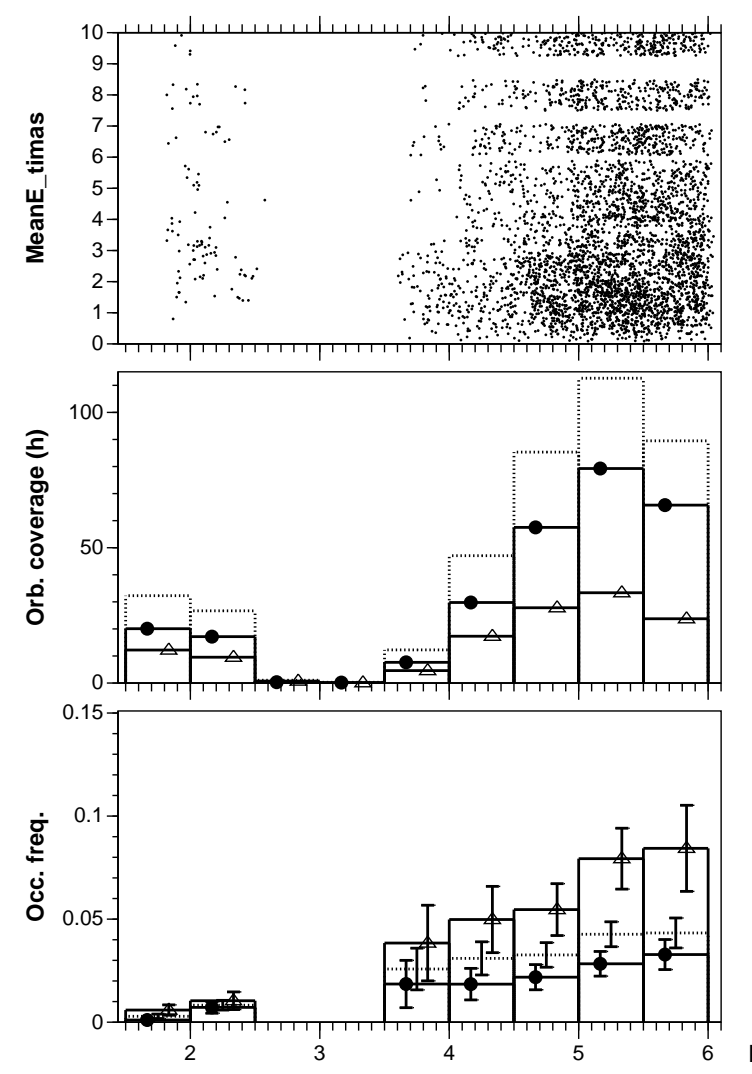
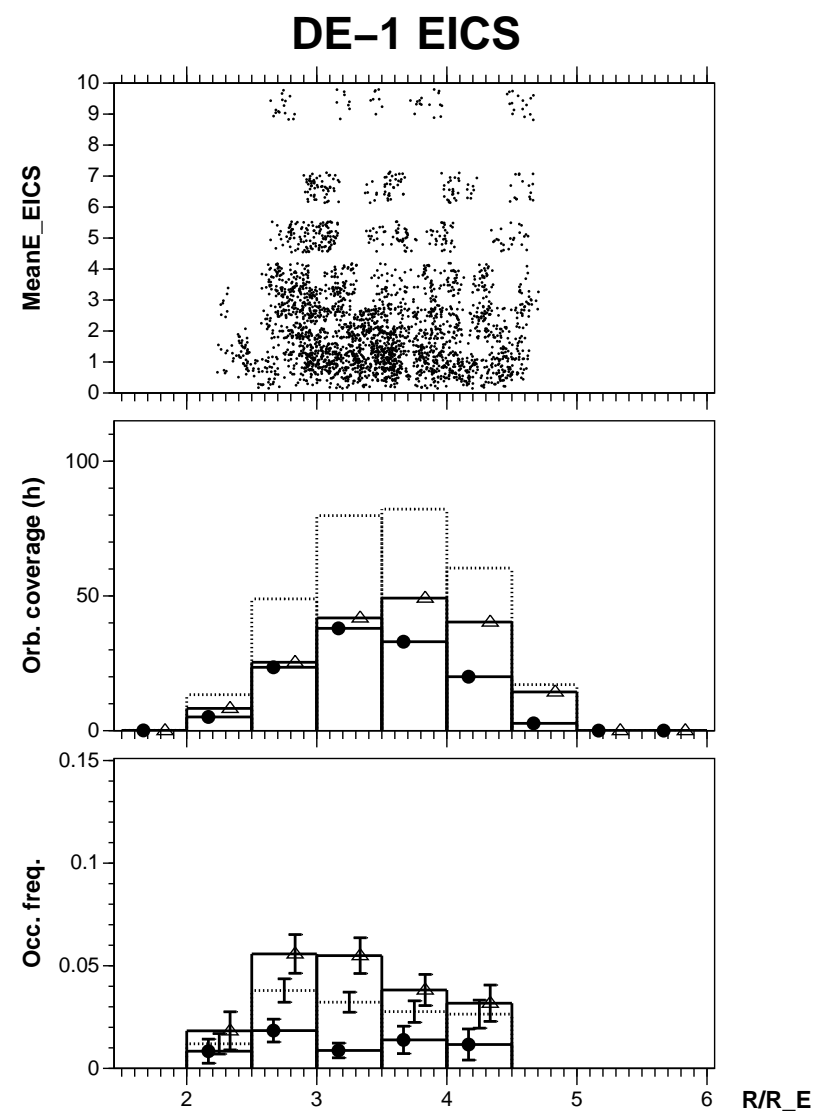

Fig. 1. Polar/TIMAS (left), DE-1/EICS (right). Top: All detected $0.5-10 \mathrm{keV}$ beams as a function of radial distance $R\left(R_{E}\right)$ and beam peak energy. Middle: Hours spent by the instrument in each bin. Bottom: Occurrence frequency of ion beams (number of points in each bin divided by the number of 12 -s samples coming from the bin, averaged over ILAT). Small $K_{p}(\leq 2)$ shown by filled circles, large $K_{p}(>2)$ by triangles. Dotted line is both $K_{p}$ s put together. All nightside MLT sectors (18-06) are included and the satellite footpoint is Sun-illuminated. The method of calculating the error bars is explained in the text. The ILAT range is 65-74.

Table 1. TIMAS and EICS high $K_{p}$ ion beam occurrence frequency when footpoint is sunlit

\begin{tabular}{llll}
\hline & MLT 18-22 & MLT 22-02 & MLT 02-06 \\
\hline TIMAS 2-2.5 $R_{E}$ & $0.016 \pm 0.01$ & - & - \\
EICS 2-2.5 $R_{E}$ & $0.022 \pm 0.011$ & - & - \\
TIMAS 4-4.5 $R_{E}$ & $0.056 \pm 0.022$ & $0.044 \pm 0.025$ & $0.016 \pm 0.014$ \\
EICS 4-4.5 $R_{E}$ & $0.058 \pm 0.025$ & $0.042 \pm 0.02$ & $0.009 \pm 0.01$ \\
\hline
\end{tabular}

energy for conditions where the satellite ionospheric footpoint is sunlit. The left panel shows TIMAS results and the right panel shows EICS. Since the peak energy is quantized to one of the detector energy channel center energies, in order to ease plotting, a uniform random number in the range $-0.5 \ldots 0.5 \mathrm{keV}$ has been added to the ordinate of each data point. Since TIMAS files have an 8 times higher time resolution than EICS, each EICS point is replicated 8 times in the upper right panel of Figs. 1 and 2 to make the number of TIMAS and EICS points mutually comparable. Comparison of the left and right panels of Fig. 1 shows that the resulting occurrence frequencies agree relatively well in those altitude bins where both instruments have coverage. Figure 2 shows the corresponding result for conditions where the satellite ionospheric footpoint is in darkness. Also, here, TIMAS and EICS ion beam occurrence frequencies are in agreement in those altitude bins where both instruments have proper coverage.

The error bars shown in all figures of this paper correspond to the standard deviations obtained by assuming that all data points within one auroral zone crossing of each bin are fully correlated. The method is as follows. For a given bin, assume there are $N$ data points, $n$ ion beams and $K$ auroral zone crossings. The occurrence frequency $f$ is given by $f=n / N$ 

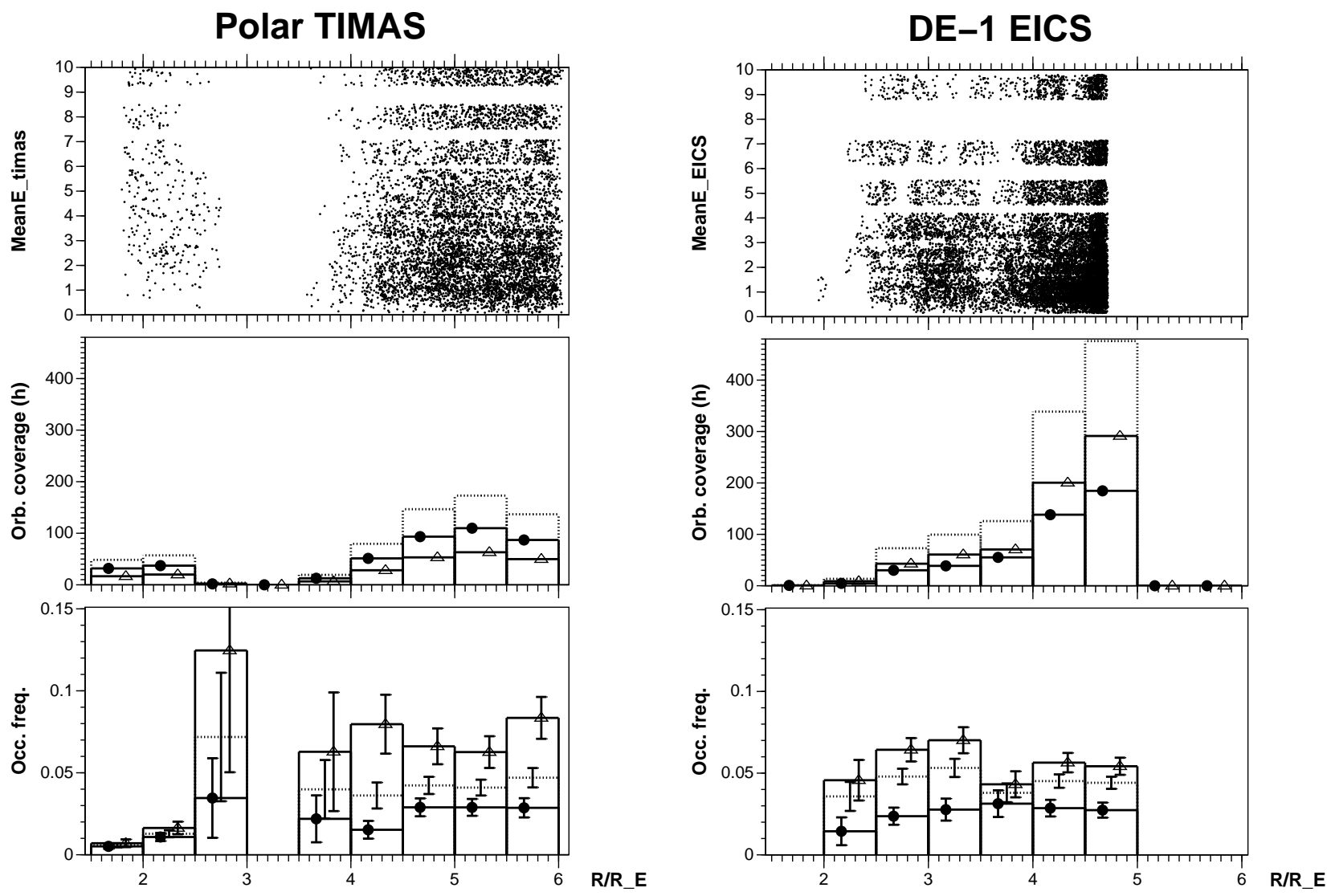

Fig. 2. Same as Fig. 1, but for conditions when the satellite footpoint is in darkness.

Table 2. TIMAS and EICS ion beam occurrence frequency when footpoint is in darkness; for high $K_{p}$ conditions, except when noted otherwise

\begin{tabular}{llll}
\hline & MLT 18-22 & MLT 22-02 & MLT 02-06 \\
\hline TIMAS 2-2.5 $R_{E}$ & $0.053 \pm 0.018$ & $0.018 \pm 0.006$ & - \\
EICS 2-2.5 $R_{E}$ & $0.044 \pm 0.012$ & - & - \\
TIMAS 4-4.5 $R_{E}$ & $0.008 \pm 0.01\left(\right.$ low $\left.K_{p}\right)$ & $0.098 \pm 0.02$ & $0.025 \pm 0.016$ \\
EICS 4-4.5 $R_{E}$ & $0.011 \pm 0.013\left(\right.$ low $\left.K_{p}\right)$ & $0.073 \pm 0.01$ & $0.03 \pm 0.008$ \\
TIMAS 4.5-5 $R_{E}$ & $0.055 \pm 0.025$ & $0.075 \pm 0.015$ & $0.016 \pm 0.007\left(\right.$ low $K_{p}$ ) \\
EICS 4.5-5 $R_{E}$ & - & $0.073 \pm 0.01$ & $0.016 \pm 0.004\left(\right.$ low $\left.K_{p}\right)$ \\
\hline
\end{tabular}

and its standard deviation $\Delta f$ by $\Delta f=\sqrt{f(1-f) / K}$. The method probably overestimates the error to some extent. This method of computing the standard deviations is applied separately in each ILAT bin, but before plotting, ILAT averaging is carried out as explained above in Sect. 2.3. Note that the standard deviation $\Delta f$ does not depend on the fact that DE-1 data points are replicated eight times, because the replication affects $n$ and $N$ but not their ratio $f$ or the number of orbital crossings $K$, and $\Delta f$ only depends on $f$ and K.

To compare TIMAS and EICS results more quantitatively we decompose the statistics in the three nightside MLT sectors $18-22,22-02$ and $02-06$. Table 1 shows the MLT- decomposed TIMAS and EICS occurrence frequencies for radial distance ranges $2-2.5 R_{E}$ and $4-4.5 R_{E}$ for sunlit cases and for $K_{p}>2$. The standard deviations are also given. Table 2 shows the corresponding comparison for darkness cases. In some cases which are noted in Table 2 we carry out the comparison for $K_{p} \leq 2$, however, because of better orbital coverage and thus smaller statistical uncertainty. From the tables we see that in cases where orbital coverage allows for comparison, the two satellites produce occurrence frequencies that in all cases are within the error limits from each other. In fact, in all cases except one, the discrepancy is clearly smaller than the standard deviation. This indicates that some points within one auroral zone crossing are in re- 


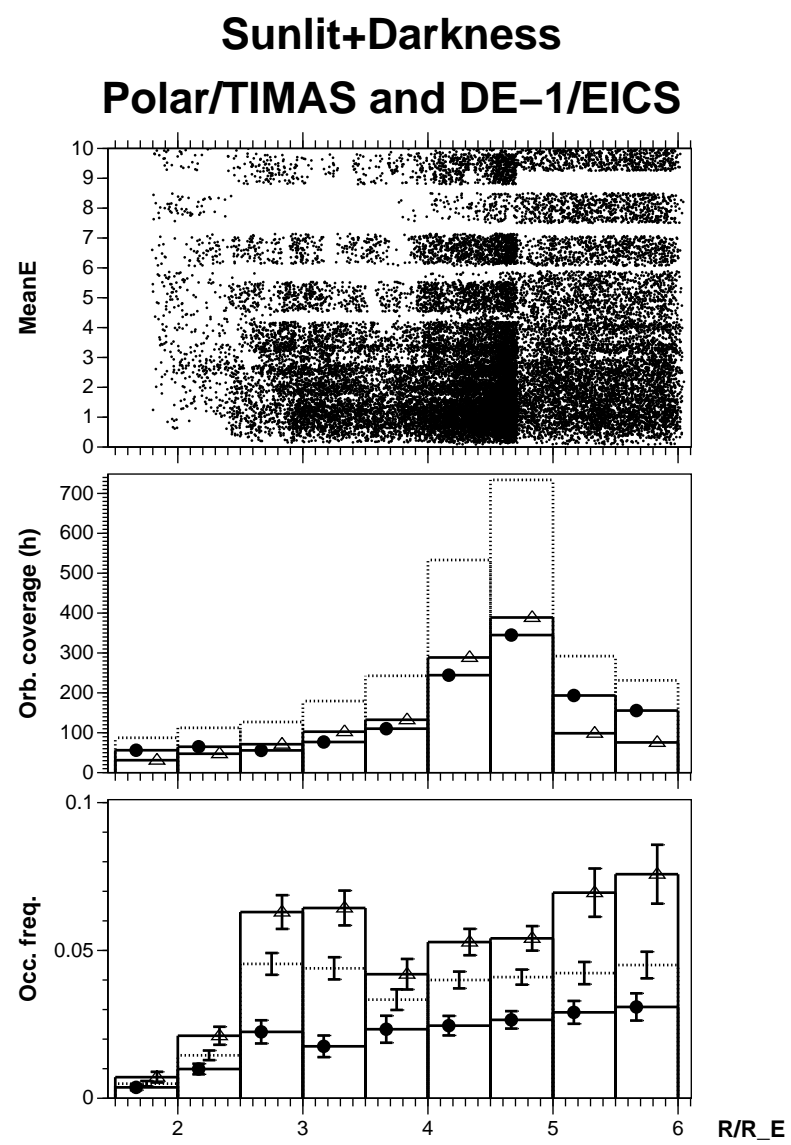

Fig. 3. Polar/TIMAS and DE-1/EICS ion beams in the nightside MLT sectors as a function of radial distance $R$. Top panel: Ion beam events (one event corresponds to $12 \mathrm{~s}$ sample) as a function of radial distance and peak energy. Middle panel: Hours spent by the instrument in each radial bin. Bottom panel: Occurrence frequency of ion beams (number of points in each bin divided by the number of samples coming from the bin). Small $K_{p}(\leq 2)$ shown by filled circles, large $K_{p}(>2)$ by triangles. Dotted line is both $K_{p}$ s put together. All nightside MLT sectors (18-06) with both sunlit and darkness conditions are included. The ILAT range is 65-74.

ality not fully correlated. Thus, the error bars shown in this paper are most probably overestimates of the true error level.

Although TIMAS and EICS agree within the error limits in the cases where they can be compared, the error limits are not very small in the intercomparison bins and thus there could, in principle, be underlying systematical differences between the two instruments that are masked by the statistical errors in the intercomparison bins that could become important later when one considers bins with smaller statistical errors. To see that this is not the case we note from Tables 1 and 2 that the differences between TIMAS and EICS are insignificantly small in nine cases out of ten, but in one case (MLT 2202, 4-4.5 $R_{E}$ radial bin), the occurrence frequencies differ by $35 \%$. A more detailed comparison shows that TIMAS in this bin disagrees not only with DE-1 in the same bin, but also with TIMAS (and DE-1) in the neighbouring bins (plots not shown). This suggests that the difference is due to statistical fluctuations rather than a systematic error. We have also manually checked the TIMAS events contributing to this bin but did not find traces of nonphysical effects that could explain why TIMAS sees 500 data points containing an ion beam in $17 \mathrm{~h}$, while it should see about 370 to make it agree with EICS.

We now discuss more quantitatively how the mutual difference between EICS and TIMAS relates to their standard deviation error estimates. If $E$ is the occurrence frequency measured with EICS and $T$ is the corresponding quantity measured with TIMAS and $\Delta E$ and $\Delta T$ are their expected standard deviations (calculated based on the number of orbital crossings as explained above), let us consider the quantity $Q=|E-T| /(0.5 \times(\Delta E+\Delta T))$, i.e. the ratio of the EICS-TIMAS discrepancy to the mean of their standard deviations. For the ten entries displayed in Tables 1 and 2, the $Q$ values are, in ascending order, 0, 0.09, 0.09, 0.16, 0.26, 0.40, $0.56,0.57,0.62$ and 1.7. All values of $Q$ are thus smaller than unity, except one (1.7), which was discussed above in the previous paragraph. The interpretation of the $Q$ values is such that, for example, $Q=0.09$ means that the standard deviation (the quantity shown as error bars in all figures of this paper) is about 10 times larger than the actual discrepancy between the two instruments. The median $Q$ value over the ten cases is 0.33 . If this value is taken as representative, it would mean that the error bars shown in this paper are overestimates by about a factor of three. This point should be kept in mind when looking at the figures, i.e. that the error bars are probably unrealistically large. For the sake of keeping the plots mathematically rigorous we elected not to divide the error bars by 3 (or some other constant). In most cases the plotted error bars do not cause problems, i.e. we can draw our conclusions even with the displayed (overestimated) error bars.

We conclude that TIMAS and EICS data agree well enough so that when the ion beams are found and the error bars are drawn in the manner described above, data from both satellites can be put in the same statistics.

\section{Results}

\subsection{Radial distance}

In Fig. 3 we show our baseline plot, which is TIMAS and EICS ion beams put together in the $0.5-10 \mathrm{keV}$ energy range for all nightside MLT sectors under sunlit and darkness conditions. The occurrence frequency of ion beams generally increases with altitude, but there is also a relative maximum in the occurrence frequency of ion beams at $3 R_{E}$ radial distance. In the baseline case (dotted line in Fig. 3 representing all $K_{p}$ s put together), the relative maximum (henceforth called "the peak") occurs at $2.75 R_{E}$ radial distance and a local minimum at $3.75 R_{E}$. The existence of a peak can be seen in earlier plots (Gorney et al., 1981; Yau et al., 1985; Kondo et al., 1990) but it has not been discussed. 


\section{$3.2 K_{p}$}

Figure 3 shows small $K_{p}(\leq 2)$ by filled circles and large $K_{p}$ $(>2)$ by triangles. The combined statistics are shown by a dotted line. Generally, the ion beam occurrence frequency is $\sim 2-3$ times larger for $K_{p}>2$ than for $K_{p} \leq 2$. The peak and the minimum above it occur at 3.25 and $3.75 R_{E}$ for large $K_{p}$, respectively. For low $K_{p}$ the peak also exists but its amplitude is weak (of the order of the error bar).

\subsection{MLT and solar illumination}

In Fig. 4 we show the ion beam statistics separated into three nightside MLT sectors in sunlit (top) and darkness (bottom) conditions. Comparing radial distance bins below $2.5 R_{E}$ for high $K_{p}$ conditions, the occurrence frequency in darkness is 0.05 , which is 2.5 times higher than in sunlit conditions in the evening sector (18-22 MLT). Overall, this is in agreement with a previous study where a ratio of $\sim 3$ was found in the occurrence frequency in darkness versus sunlit beams (Collin et al., 1998), although the beam selection criteria and thus the absolute occurrence frequencies are different in the two studies. It is also seen that in the evening sector between 2.5 and $3.5 R_{E}$ the occurrence rate of ion beams is higher during darkness than during sunlit conditions, although the difference is not as large as below $2.5 R_{E}$. In the midnight (22-02) sector there is a pronounced peak at the $2.5-3 R_{E}$ bin in sunlit conditions, but its reliability is questionable due to the large error bars. In midnight darkness conditions the peak is less pronounced than in the evening sector, especially for high $K_{p}$. High $K_{p}$ midnight is the combination that is most affected by substorms, so substorms are a likely candidate for causing the difference between the midnight and the evening sectors in this respect. In a recent study of auroral cavities the midnight MLT sector was also found to have different behaviour which was attributed to substorms (Janhunen et al., 2002). In the morning sector ion beams are rare.

The relative peak in occurrence frequency around $3 R_{E}$ radial distance is most apparent in the evening sector (1822 MLT). In this sector we also have the best overall orbital coverage, so that the statistical errors are the smallest. In the morning sector there is no orbital coverage at all close to $3 R_{E}$ so the existence of the peak remains uncertain in that MLT sector.

Ion beams for small $K_{p}$ are rare in other MLT sectors except the midnight sector, where they are almost equally common for small and large $K_{p}$ indices (except in the midnight 2.5-3 $R_{E}$ bin under sunlit conditions, which, however has rather large error bars as we remarked above).

\subsection{Solar cycle effects}

In Fig. 5 we show the statistics separated into solar minimum (left panel) and solar maximum years (right panel). We take years 1978-1982, 1988-1992 and 1999-2003 to be solar maximum years. Other years are taken to be solar minimum. Thus, TIMAS (1996-1998) contributes to the solar minimum statistics only. There is hardly any systematic dependence on the solar cycle. From the orbital coverage panels one sees that during the solar maximum years, high $K_{p}$ values are always more common than small $K_{p}$ values, while for solar minimum years they are about equally common, which is not surprising.

\subsection{ILAT dependence}

To investigate ILAT dependence, we show in Fig. 6 the occurrence frequency for all nightside MLT but decomposed into three ILAT ranges: 65-68 (bottom panel), 68-71 (middle panel) and 71-74 (top panel). We see that small $K_{p}$ events occur only seldom below ILAT 68 , which is natural. The occurrence frequency is largest in the 68-71 ILAT bin, which corresponds to the average auroral oval latitudes. For high $K_{p}$, the peak is seen to exist at all ILAT ranges separately.

Another view on the ILAT dependence is provided in Fig. 7, which shows the two-dimensional ILAT-altitude statistics of all nightside ion beams.

In Figs. 8 and 9 we show that statistics in the MLT-ILAT and MLT- $R$ planes, respectively. In the MLT-ILAT plane the auroral dependence of ion beams is clearly seen, i.e. that beams occur at lowest ILAT close to midnight and that they occur most often near midnight, with a slight preference for pre-midnight. In Fig. 9 the auroral dependence can be seen as well, together with the fact that the $\sim 3 R_{E}$ occurrence frequency peak is visible in essentially all MLT sectors (16-04) where there is orbital coverage and ion beams.

From Fig. 8 one sees that ion beams, when all altitudes are put together, occur mostly in the 22-24 MLT sector, in accordance with the results of Yau et al. (1985) and Gorney et al. (1985), whereas Johnson (1983) in his Fig. 5 found that ion beams, at least during disturbed conditions, occur mostly in the 15-21 MLT range. The results of Johnson (1983) represent the altitude range $6000-8000 \mathrm{~km}$ only, however, which corresponds to our second altitude bin $\left(2-2.5 R_{E}\right.$ radial distance). Inspection of Fig. 4 shows that in this altitude bin, ion beams for large $K_{p}$ are indeed much more common in the 18-22 than in the 22-02 MLT sector in our database. Thus, our results are also in agreement with those of Johnson (1983). Thus, at low altitude and high $K_{p}$, ion beams are mainly an evening sector phenomenon, while at high altitude and low $K_{p}$ they are more a midnight sector phenomenon.

\subsection{Ion beam energy, particle flux and ion energies}

Thus far we have only considered the occurrence frequency of ion beams, but now we will study the energy and particle fluxes carried by the beams, as well as the mean energy for the evening sector. We limit ourselves to the evening sector in this substudy, because in the morning sector we have only a few ion beams, as seen above, and in the midnight sector the situation is somewhat more complicated (see below). In Fig. 10, left panel, we show the invariant energy flux in $\mathrm{mW} \mathrm{m}^{-2}$ (panel c) and the invariant particles flux in 


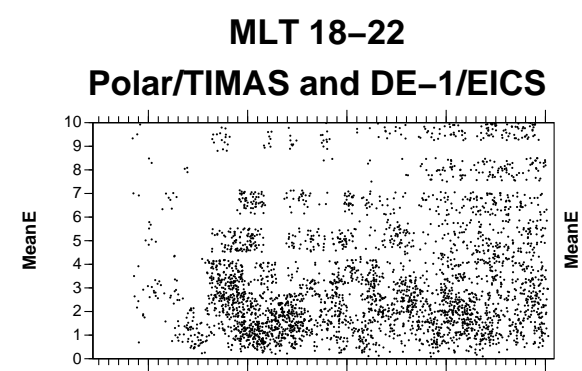

\section{MLT 22-02 \\ Polar/TIMAS and DE-1 EICS}
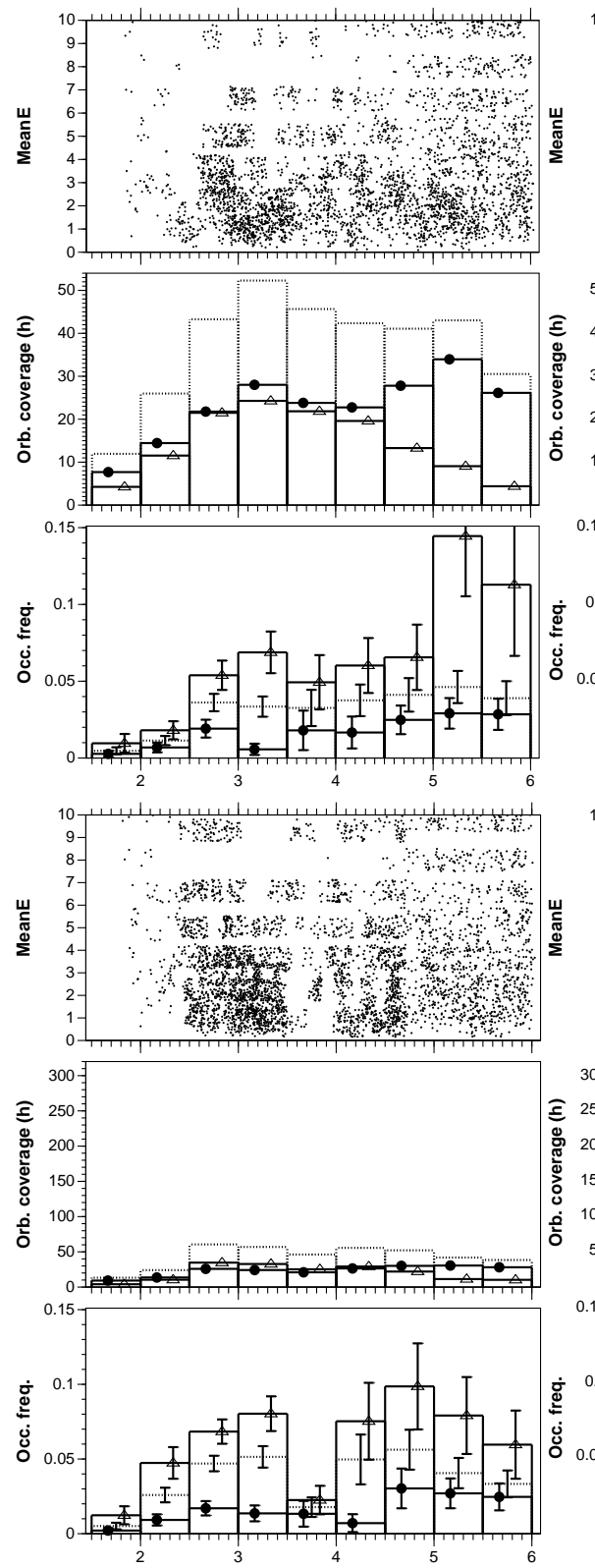
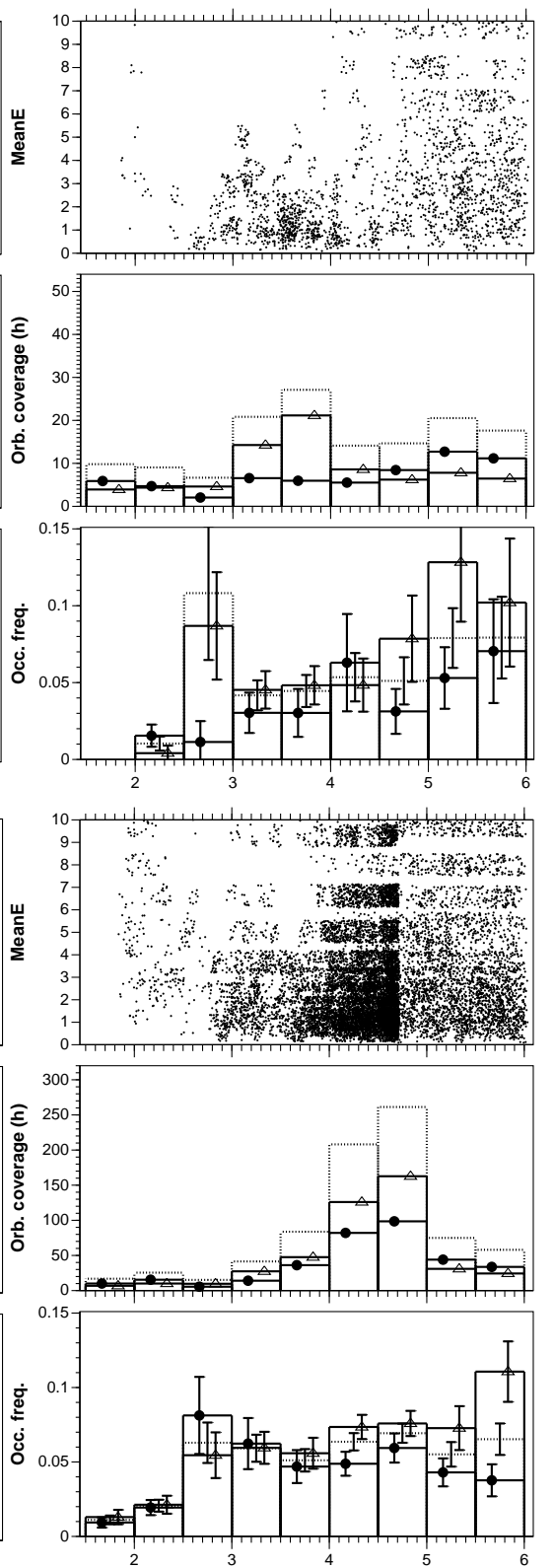

MLT 02-06 Polar/TIMAS and DE-1/EICS
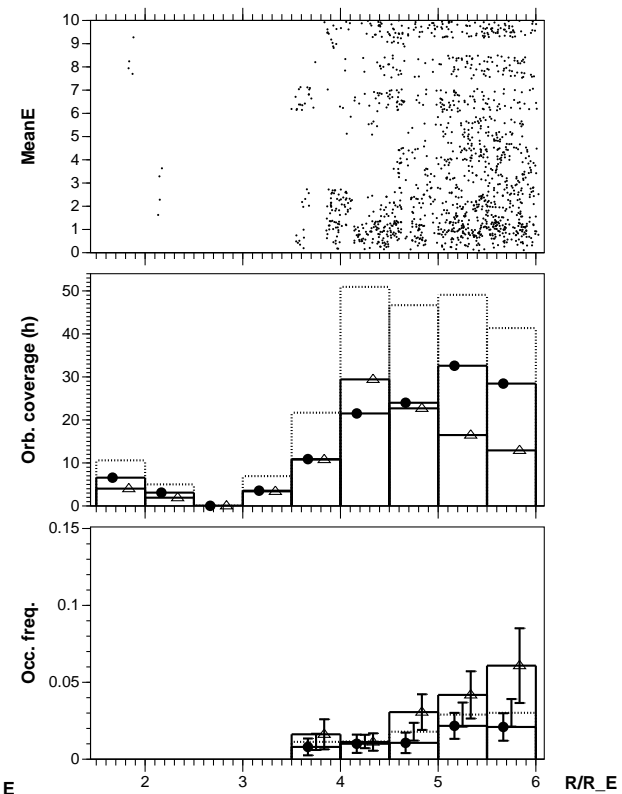

R/R_E
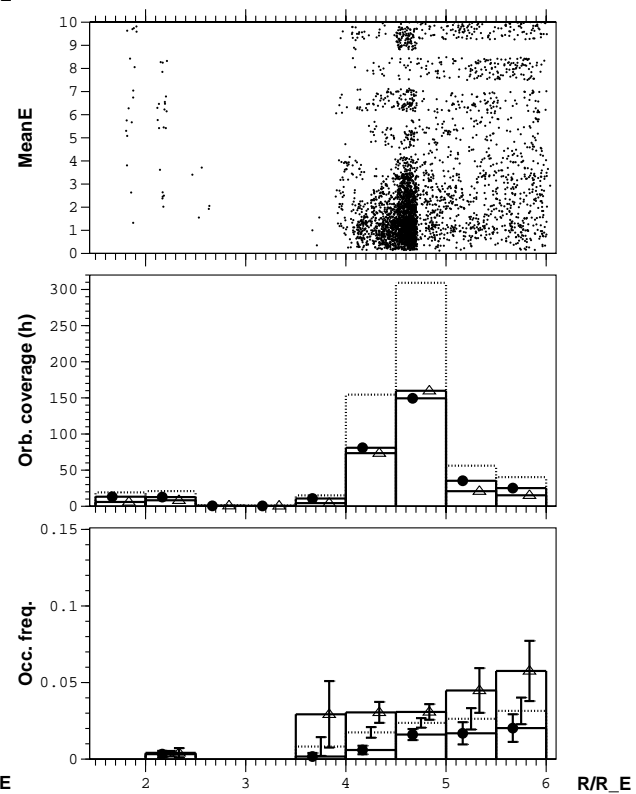

Fig. 4. Same as Fig. 3, but separated in the three nightside MLT sectors with sunlit (top) and darkness (bottom) conditions.

$\mathrm{m}^{-2} \mathrm{~s}^{-1}$ carried by the upward ion beams (panel d). Both quantities are altitude-invariant in the sense that they have been projected to the ionospheric plane by multiplying them by the magnetic field ratio. They have been averaged linearly over all data points. Regions where no ion beams are detected are assumed to have zero energy and particle flux, thus, the quantities plotted tell how much energy and particle flux is carried away from the ionosphere globally by ion beams, on average, in the 68-71 ILAT range and 18-22 MLT.

If all ion beams could be reliably detected and if there were no wave-particle interactions, the conservation of beam particles would dictate that the invariant particle flux should be independent of altitude, if the external conditions (magnetic activity level, solar illumination condition, etc.) are also the same for all altitudes. From panel (d) of Fig. 10, left panel, we see that this is clearly not the case, as the invariant number flux generally varies with altitude. Thus, if we exclude instrumental effects for a moment (they will be discussed later in this part and in the Discussion section below), it must be that beams in some altitude regions turn at least temporarily into something which is not recognizable as a beam any longer (e.g. ion conics). In other regions where the invariant particle flux increases with altitude it must happen that the conics turn back into beams (probably by the mirror force) 


\section{Solar minimum}

\section{Polar/TIMAS and DE-1/EICS}
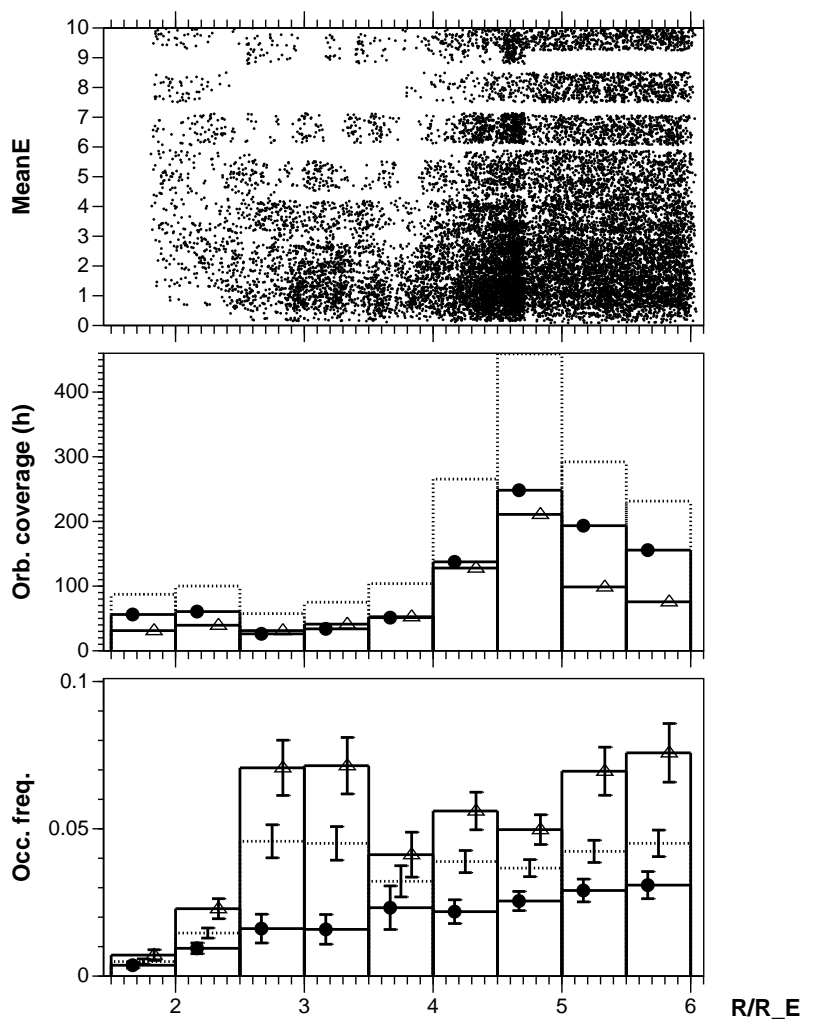

Solar maximum DE-1/EICS


Fig. 5. Same as Fig. 3, but separated for solar minimum (left) and solar maximum years (right). All nightside MLT sectors with both sunlit and darkness conditions are included, and the ILAT range is 65-74.

or that new particles enter pre-existing beams or new beams are initiated at the altitude in question.

In Fig. 10, the invariant energy flux (panel c) and particle flux (panel d) altitude variation follow the variation of the occurrence frequency of ion beams (panel b). If the only energization mechanism were a potential drop acceleration below $8000 \mathrm{~km}$, for instance, all three quantities should stay constant above $8000 \mathrm{~km}\left(R=2.25 R_{E}\right)$. As seen from Fig. 10, this is not the case, but all three quantities drop rather dramatically at $R=3.75 R_{E}$ for high $K_{p}$ (for small $K_{p}$, the drop occurs at 3.25). At higher altitudes they slowly recover. This suggests that some process operating at the altitude where the quantities drop is making the ion beams at least temporarily unrecognizable.

The last panel (panel e) of Fig. 10 is the mean beam energy (the mean invariant energy flux divided by the mean invariant particle flux). We will discuss this panel below, after presenting a simple Monte Carlo simulation.

Here, we discussed only the evening sector. Similar trends in the invariant energy, particle fluxes, and ion mean energy exist in the midnight sector for low $K_{p}$ indices, but not for high $K_{p}$. A probable reason for why the high $K_{p}$ midnight sector behaves differently in this regard is that data in the midnight sector during high $K_{p}$ are probably dominated by a direct influence of substorm phenomena.

\subsection{Monte Carlo simulation of ion beams}

A physical understanding of the altitude behavior of all the quantities shown in the left panel of Fig. 10 is not easy without some computer modelling. To this end we present a simple Monte Carlo simulation in which we generate 10000 ion beams. The invariant particle flux of each ion beam is a random number selected from the exponential distribution (Press et al., 1992), with mean $5.44 \times 10^{11} \mathrm{~m}^{-2} \mathrm{~s}^{-1}$. The specific numerical constants have been selected to produce results that resemble those in the left panel of Fig. 10. The ion beam is assumed to have zero energy at the bottom of the acceleration region. The acceleration region is modelled by either an open or closed potential structure, whose depth is another exponentially distributed random number with mean $V_{0}=2 \mathrm{kV}$. The potential structure $V(R)$ is defined by $V(R)=V_{0} \exp \left(-\left(\left(R-R_{0}\right) / \Delta R\right)^{2}\right)$ in the closed case. Here, $R$ is the radial distance in units of $R_{E}$ and $R_{0}=\left(R_{1}+R_{2}\right) / 2$ and $\Delta R=\left(R_{2}-R_{1}\right) / 2$ are parameters defining the center and width of the potential structure. The parameter $R_{1}$ de- 
Sunlit + Darkness

\section{Polar/TIMAS and DE-1/EICS}
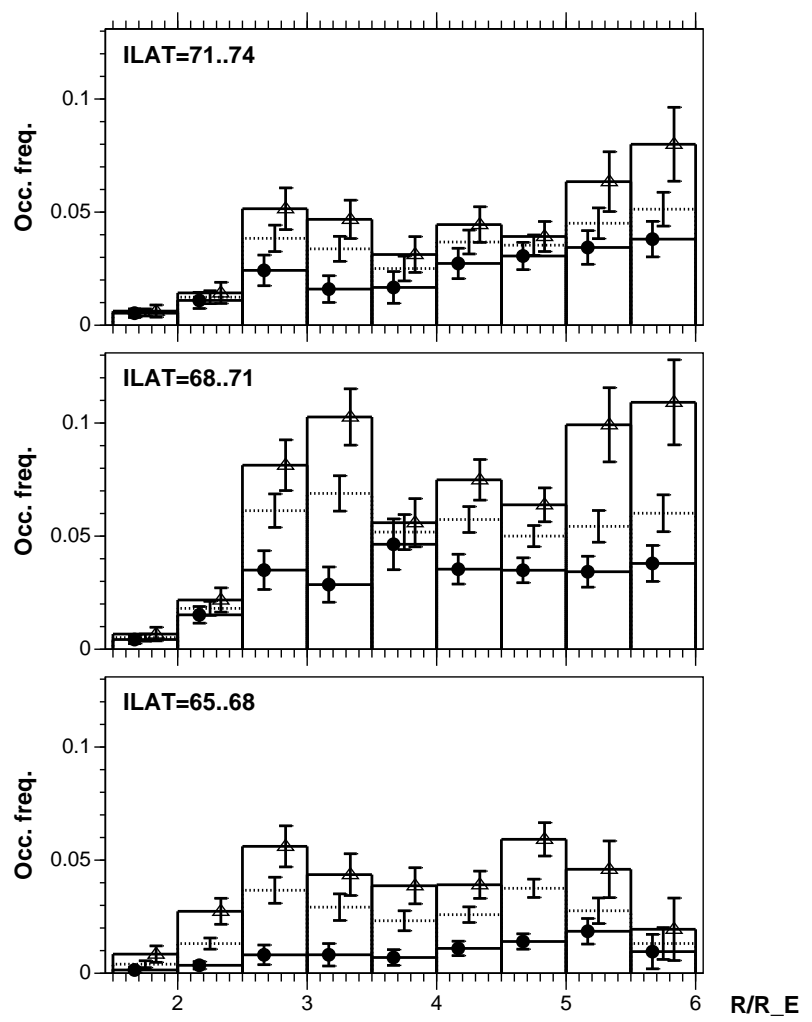

Fig. 6. Same as the bottom panel of Fig. 3, but shown separately for the ILAT ranges 71-74 (top), 68-71 (middle) and 65-68 (bottom). All nightside MLT sectors (18-06) are put together, and both sunlit and darkness conditions are included.

pends on $V_{0}$, so that $R_{1}=\max \left(2.5-V_{0} / 5,1.5\right)$ while $R_{2}$ has the constant value 3.5. The parameters $R_{1}$ and $R_{2}$ represent the bottom and top altitudes of the potential structure, respectively, and their values have been selected to be physically reasonable (Janhunen and Olsson, 2002). The open potential structure case is obtained by setting $V(R)$ to $V_{0}$ when $R>R_{0}$. The simulated ion beams are detected by many virtual instruments placed at different altitudes, using the same thresholds as are used when processing TIMAS and EICS data.

We now discuss an instrumental effect related to satellite speed. Due to the fast velocity at low altitude, the satellite traverses a rather long ILAT distance during one measurement cycle (12 s for Polar, $96 \mathrm{~s}$ for DE-1; we use $12 \mathrm{~s}$ in the simulation since the low-altitude data come from Polar). At the lowest altitude bin the north-south distance traversed during $12 \mathrm{~s}$ by Polar is about $24 \mathrm{~km}$ in the ionosphere, which is a longer distance than the width of narrow auroral arcs. Thus, the measured ion beams will be effectively averaged with background, which reduces their observed intensity (the particle and energy flux). However, the probability that the satellite hits an ion beam during $12 \mathrm{~s}$ is correspond-

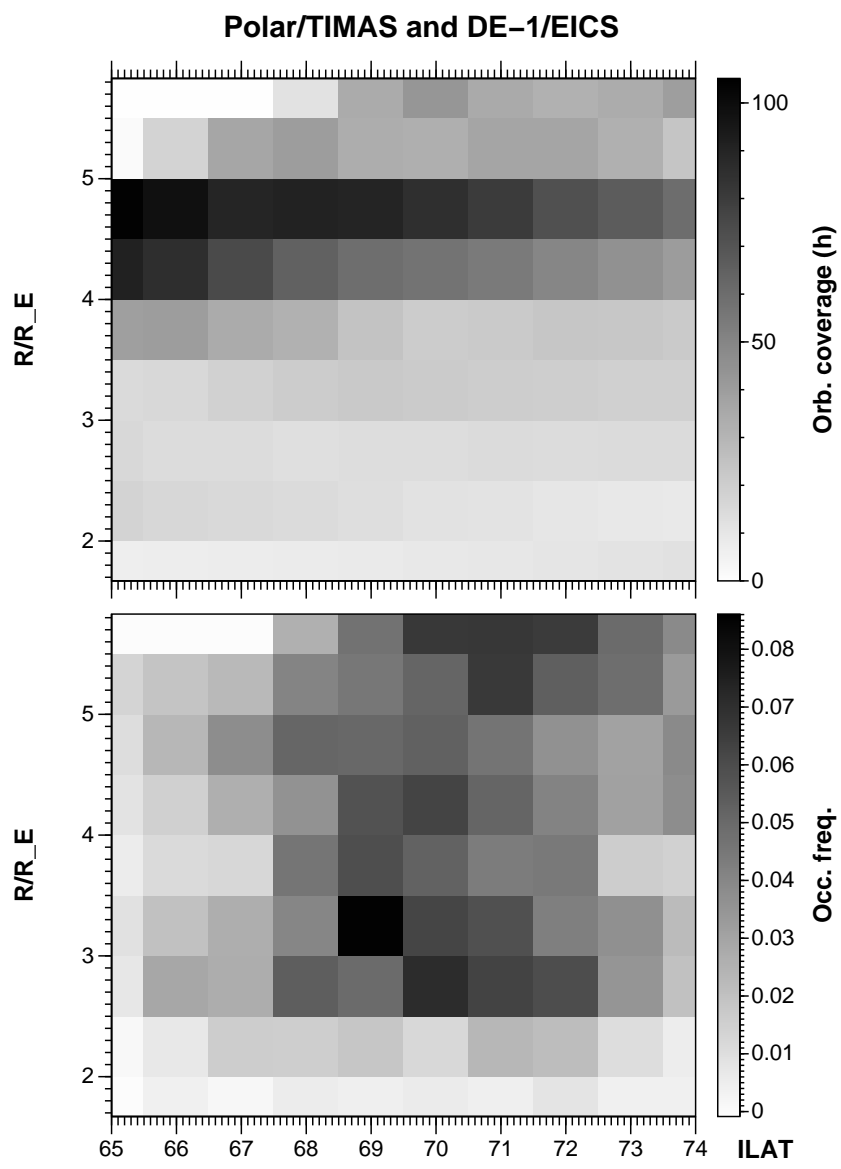

Fig. 7. Top: Orbital coverage in hours in each bin. Bottom: Occurrence frequency of all nightside $0.5-10 \mathrm{keV}$ ion beams as a function of ILAT and radial distance $R$. Both TIMAS and EICS data are put together, as are all $K_{p}$ values, all nightside MLT sectors (18-06), and sunlit and darkness conditions.

ingly higher. Thus, there are two competing effects, the first decreasing and the second increasing the probability of detecting ion beams at low altitude. Since the first effect is exponential and the second one is only linear, the net effect is a reduction of the probability of detecting beams. We model both of these satellite speed related effects by using a realistic satellite velocity altitude dependence profile.

In the simulation we assume that the average width of ion beams' regions when mapped to the ionosphere is $8 \mathrm{~km}$. The widths of the ion beams should match the typical widths of inverted-V regions and optical auroral arcs. The widths of inverted- $\mathrm{V}$ regions and optical arcs match each other when compared with compatible criteria (Stenbaek-Nielsen et al., 1998). The widths of optical arcs vary, but $8 \mathrm{~km}$ is a typical value.

Optionally, uniform wave energization (constant energy increment in each altitude step) is added into the simulation. The wave energization $W(R)$ is taken to be proportional to $V_{0}$ and taken to start from $R=2$, so that $W(R)=$ $0.2 e V_{0} \max (R-2,0)$. When wave energization is on, the ion beam energy $E$ is calculated from $E=e V(R)+W(R)$, 


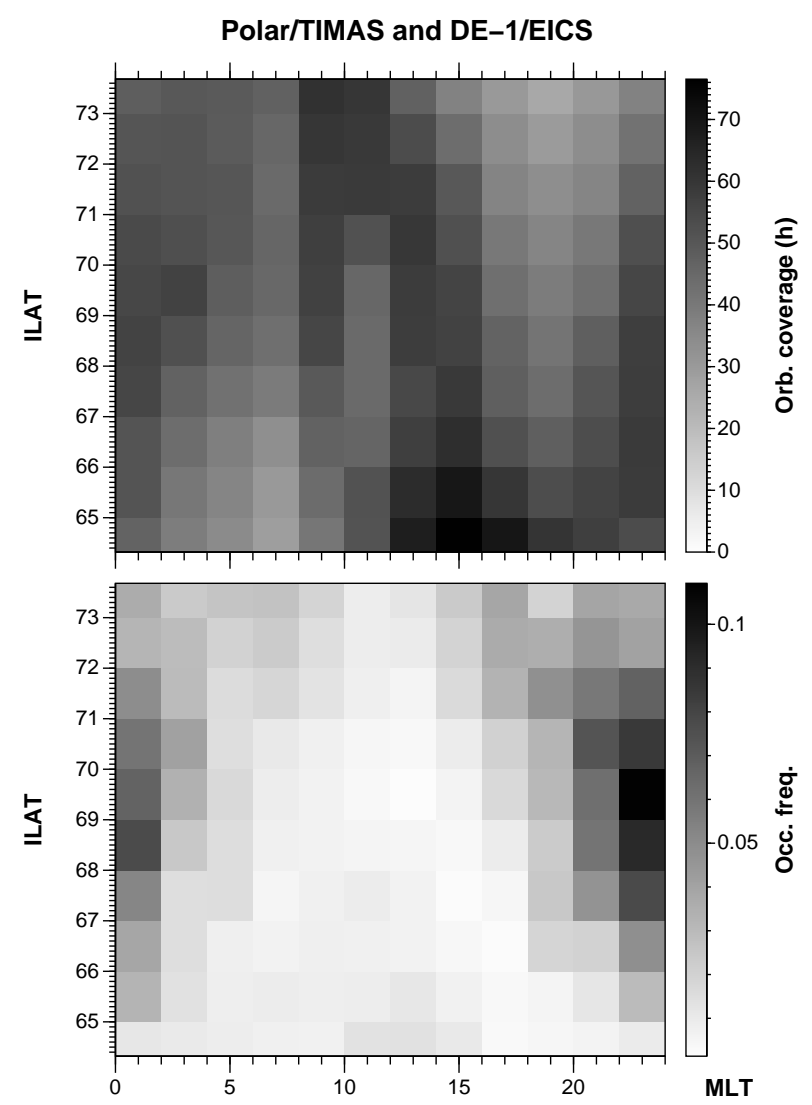

Fig. 8. Top: Orbital coverage in hours in each bin. Bottom: Occurrence frequency of all $0.5-10 \mathrm{keV}$ ion beams as a function of MLT and ILAT for all radial distances smaller than $6 R_{E}$. All $K_{p}$ values, all radial bins and data from both TIMAS and EICS are put together. The dayside MLT range 10-14 may contain data errors and should not be looked at.

otherwise, from $E=e V(R)$. The invariant energy flux is simply the invariant particle flux multiplied by $E$.

The right panel of Fig. 10 shows four cases: closed potential without wave energization (solid line), closed potential with wave energization (solid line with black circles), open potential without waves (dotted line), and open potential with waves (dotted line with black circles). We see that the model with a closed potential and wave energization corresponds to the left panel of Fig. 10 quite well, while the other models have severe problems in reproducing some of the features in the data.

Intuitively, one could expect that the mean energy of ions should increase when the ions move inside a potential structure and are speeded up. Looking at Fig. 10 (panel e), this is not the case, however, but rather there is a decrease. The main reasons are the satellite speed dependent effects discussed above. The reason why there is a slight peak at $R=2 R_{E}$ in the mean energy (Fig. 10, panel e), even in the U-potential curves (dotted lines), is that at $R<2 R_{E}$ part of the potential drop is still above the satellite, so the mean energy is smaller for that reason and at $R=2 R_{E}$, only the strongest beams are detectable due to the satellite

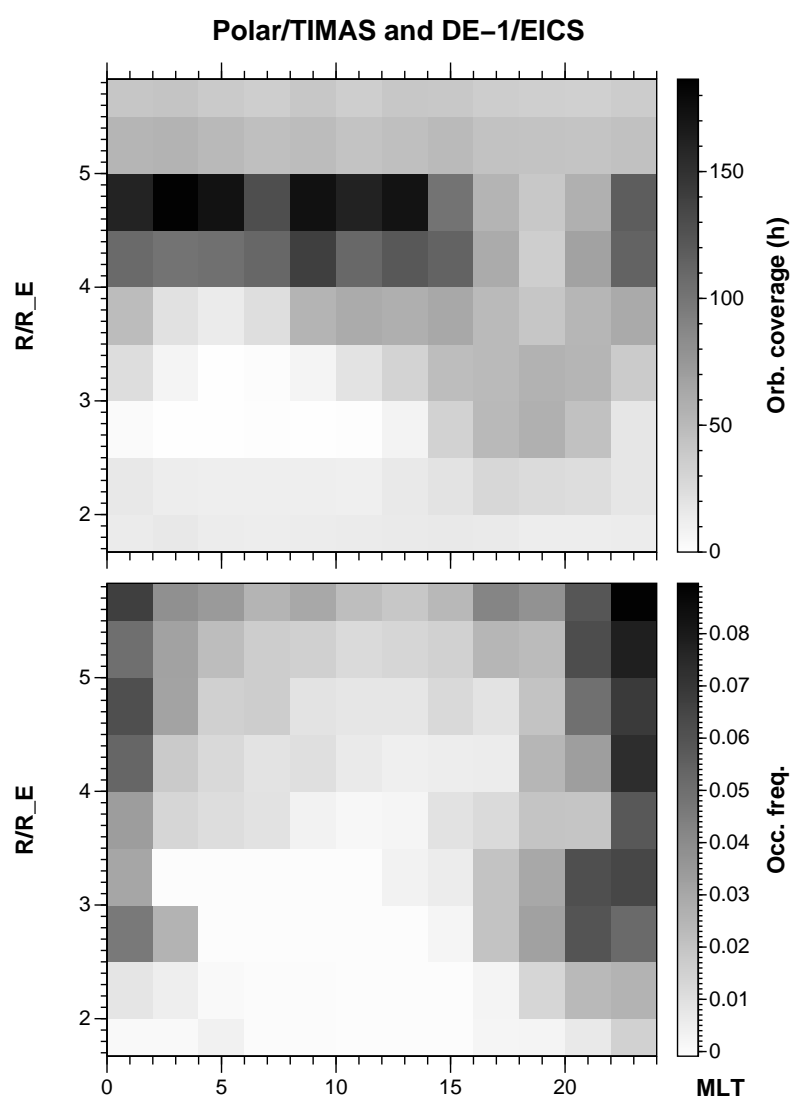

Fig. 9. Top: Orbital coverage in hours in each bin. Bottom: Occurrence frequency of all $0.5-10 \mathrm{keV}$ ion beams as a function of MLT and radial distance $R$ for all ILAT in the range 65...74. Both TIMAS and EICS data are put together, as are all $K_{p}$ values, with sunlit and darkness conditions. The dayside MLT range 10-14 may contain data errors and should not be looked at.

speed smearing-out effect and thus, the high energy beams are overrepresented.

We verified that the results do not change much if the details of the potential structure bottom altitude dependence are changed or replaced by random numbers. To achieve realistic results it is only of importance that some variations of the potential structure bottom altitude occur. Such variations can be caused by the seasonal dependence, for example.

\section{Discussion}

In this study we have confirmed previous results, such as the fact that ion beams occur less often in the morning sector than in the evening and midnight sectors and that low altitude beams $\left(<2.5 R_{E}\right.$ ) occur more often when the ionosphere is in darkness than when it is illuminated by the Sun. Also, the result that ion beam occurrence frequency increases with increasing $K_{p}$ index is in accordance with previous studies.

The most interesting result is that there is a peak in occurrence frequency at $\sim 3 R_{E}$ radial distance. (Equivalently, one could also speak of a dip in occurrence frequency at around 


\section{Sunlit + Darkness}

MLT 18-22, ILAT 68-71

\section{Polar/TIMAS and DE-1/EICS}
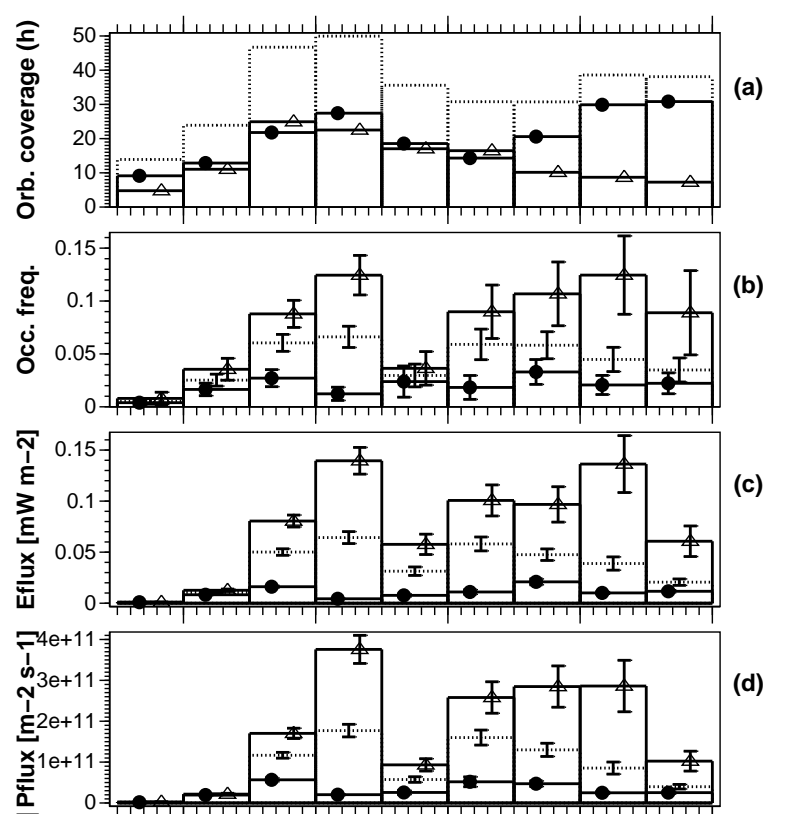

(d)

(b)

(c)

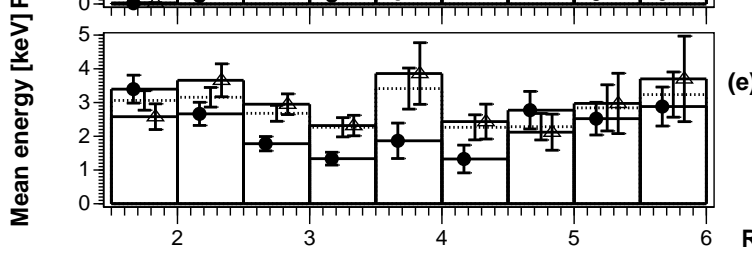

(e)
R/R_E
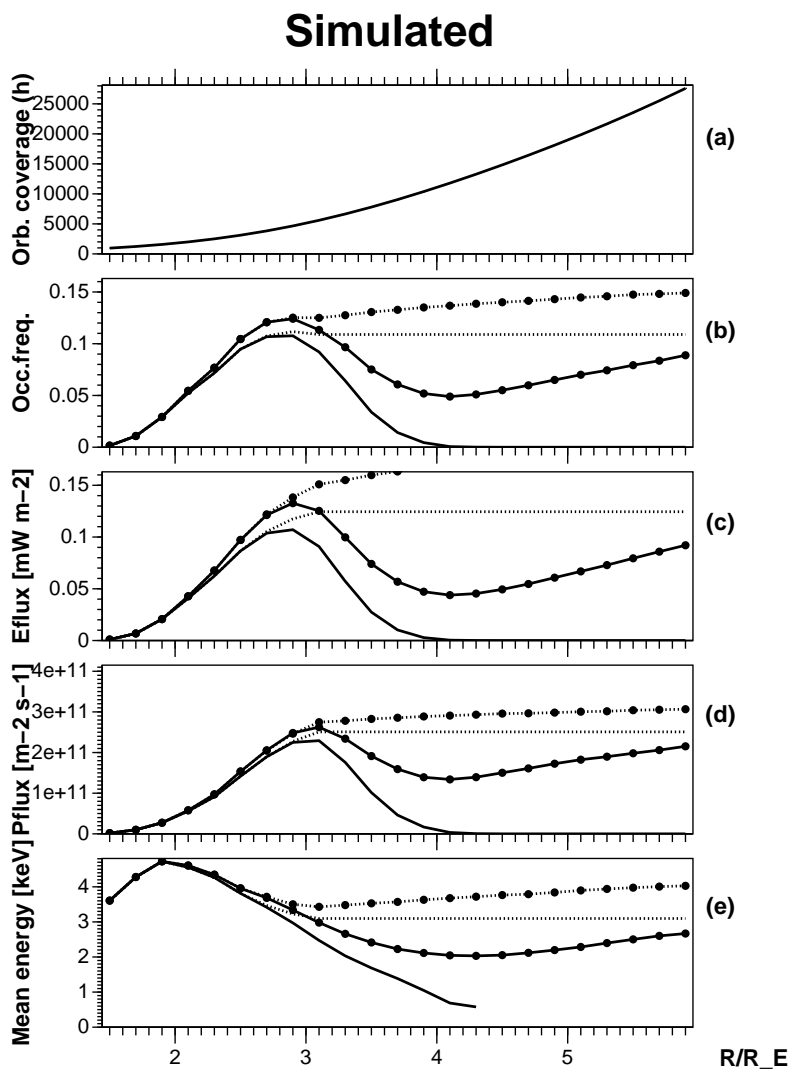

Fig. 10. Left panel: Mean energy and particle fluxes carried by ion beams in the evening MLT sector (18-22) and ILAT range 68-71. (a) Orbital coverage in hours, (b) occurrence frequency of ion beams, (c) average invariant energy flux ("invariant" means the quantity is projected to ionospheric altitude), (d) average invariant particle flux, (e) mean energy (panel c divided by panel d). Right panel: Four simulated examples (see text), solid line corresponds to a closed potential structure and dotted line to an open structure; lines with black circles correspond to cases having additional wave energization (see text).

$3.75 R_{E}$.) The peak can also be seen as an enhancement in invariant energy and particle fluxes. A peak in occurrence frequency can be seen in earlier plots (Yau et al., 1984, Fig. 4, right panel; Kondo, 1990, Fig. 7 right panel; Peterson et al., 1992, Fig. 2, top left panel), but it has not been investigated more closely. These earlier studies use DE-1 data, which, in our case, is the only contributor at the peak altitude, so the earlier results are in this sense not independent from ours, although our use of Polar data in this study has allowed us to validate DE-1 data at low and high altitude. Another result is an increase in ion beam occurrence frequency for $R>4 R_{E}$; it is not possible to obtain this result with DE-1 alone because DE-1 apogee radial distance is $4.5 R_{E}$. We will now itemize our main results:

1. In the baseline case (all MLT, ILAT, $K_{p}$ and solar illuminations conditions put together), a peak in occurrence frequency of ion beams exists at $2.75 R_{E}$ radial distance, and a local minimum occurrence frequency above it at $3.75 R_{E}$.

2. When considering low and high $K_{p}$ separately, the solar cycle does not have a notable influence on the ion beam occurrence frequency.

3. In the evening sector, a peak is also present in the invariant energy and particle fluxes carried by upward ion beams, where "invariant" means that both quantities are projected to the ionospheric plane. In the midnight sector (plots not shown) there is a peak for low $K_{p}$, but not for high $K_{p}$; the high $K_{p}$ statistics in the midnight sector is probably dominated by a direct influence of substorms, which makes the situation more complicated.

4. The peak appears in all ILAT ranges, but is most visible in the 68-71 ILAT bin, probably because it corresponds to the average auroral oval latitude.

5. At low altitude $\left(R<2.5 R_{E}\right)$ and high $K_{p}$, ion beams are mainly an evening sector phenomenon, while at high 
altitude ( $R>3 R_{E}$, say) and low $K_{p}$ they are more a midnight sector phenomenon. The reasons remain unknown, but need further investigation. The result suggests that ion beams in the midnight sector do not directly come from the region $2.5 R_{E}$, rather they are the result of processes acting at intermediate altitudes.

6. Our simple Monte Carlo simulation demonstrates that a closed potential structure with some wave energization can explain the altitude dependence of the ion beam occurrence frequency, energy flux, particle flux and mean energy in the evening sector (Fig. 10).

Since the $\sim 3 R_{E}$ peak (or, equivalently, the dip above it) is the main result of the paper, it is appropriate to try to also list the possible instrumental explanations for it. We now discuss three such instrumental explanation attempts for the peak seen in the occurrence frequency of ion beams and rule them out one by one:

1. The flux tube scaling dictates that the number flux of an ion beam with ionospheric origin will decay as $R^{-3}$. This implies that weak beams fall below the instrument threshold from some altitude upwards. However, the mapped-to-ionosphere instrument threshold for both Polar/TIMAS and DE-1/EICS is about ten times smaller, even at the highest altitude $\left(6 R_{E}\right)$ and highest energy $(10 \mathrm{keV})$ than our imposed invariant energy flux threshold of $0.2 \mathrm{~mW} \mathrm{~m}^{-2}$, so the flux tube scaling effect does not play any role in our study. We also checked with the Monte Carlo simulation that, although the flux tube scaling effect can, if one assumes unrealistic instrument threshold values, produce a peak and a subsequent smooth depression, it can never produce the observed subsequent increase at higher altitudes.

2. The peak does not disappear when the data are studied separately for different geomagnetic disturbance levels ( $K_{p}$ index), solar cycle phase, solar illumination condition, magnetic local time and invariant latitude. This rules out the possibility that the peak could be due to a correlated time/altitude orbital characteristic, together with some instrumental problem that persisted in a certain time period.

3. As pointed out in more detail in Sect. 3.7 above, satellite speed related effects decrease the probability of seeing ion beams at low altitude. This effect does not, however, explain the existence of the peak.

We will now discuss possible physical mechanisms responsible for the peak in the occurrence frequency of ion beams and thereafter, the high-altitude ion beam behavior.

The possibility for a downward field in the 15000$20000 \mathrm{~km}$ altitude range has been invoked as one of the possibilities when trying to understand counterstreaming ions (Sagawa et al., 1987; Horita et al., 1987). Later, a downward electric field in the $3-4 R_{E}$ radial range has also been proposed as an explanation for the lack of auroral potential structures above $4 R_{E}$ radial distance (Janhunen et al., 1999; Janhunen and Olsson, 2000). Our Monte Carlo simulations demonstrated (Sect. 3.7 above) that indeed, a closed potential structure is able to explain the occurrence frequency peak of the ion beams.

Beam ions are likely to be energized by both auroral potential structures and waves. This is already evident from the fact that one often sees ion beams of $10-20 \mathrm{keV}$ energy in TIMAS data (even energies as high as $40 \mathrm{keV}$ have been reported, Lundin and Eliasson, 1991), while potential structures of comparable magnitude are rare (Olsson et al., 1998). Also, the fact that the ion beam occurrence frequency continues to increase at high altitudes $\left(R>4 R_{E}\right)$ suggests that there is continuous wave-induced ion heating or parallel electric fields at high altitude.

Indeed, to explain the increase in the ion beam occurrence frequency at high altitude quantitatively, some wave energization was necessary to insert in the Monte Carlo simulation of Sect. 3.7. Other explanations (in particular, those that involve an open potential structure or no significant potential structure at all) might perhaps be constructed, but they should by necessity include rather strongly altitude-dependent wave heating or some other altitude-dependent process not included in our model.

Even when the wave amplitudes are altitude-independent, the efficiency of perpendicular wave heating should be inversely proportional to the ion beam velocity, because the wave heating per unit altitude interval is proportional to the time the particles spend within the interval. Coming back to the Monte Carlo model, the ion beams, therefore, slow down where there is downward electric field and thus, the perpendicular heating (energization) should be locally enhanced. The result could be that some of the beams turn into ion conics. Ion conics are indeed observed at $\sim 3 R_{E}$ radial distance (Klumpar et al., 1984), where the slowing down of the beam occurs in our model. At higher altitude the mirror force would turn them into beams again as the energy obtained from the waves would be gradually turned from perpendicular to parallel kinetic energy. An analogous process operating in the return current region below $7000 \mathrm{~km}$ altitude has been discussed earlier by Gorney et al. (1985) and has recently been confirmed by FAST observations (Ergun et al., 1998). This additional effect was not included in the Monte Carlo simulation, but if included, it could make the dip above the occurrence frequency peak stronger than what the simulation produced, thus perhaps further increasing the agreement with data (Fig. 10, left panel).

Upward parallel electric fields at high $\left(R>4 R_{E}\right)$ altitude could, in principle, also be a possibility to explain ion beam energization at that altitude range, but this explanation is rather unlikely as we are not aware of inverted-V electron signatures at that altitude, at least not with $\mathrm{keV}$ energies. If such a structure would exist, it should occur in combination with a closed potential structure at lower altitude, in order to explain the peak.

More statistical studies of the altitude dependence of parameters other than ion beams (auroral potential structures, 
density cavities (Janhunen et al., 2002), waves, and electron anisotropies) are completed or in progress by us and may help resolve the question of the potential structures and their associated wave-particle interactions more fully.

No matter what the processes are that explain the peculiar behavior at $\sim 3 R_{E}$ radial distance, it is clear that there is a nontrivial and relatively narrow altitude range around $3-4 R_{E}$ radial distance that seems thus far to have received too little attention in auroral physics.

Acknowledgements. Acknowledgements. The work of AO is supported by the Swedish Research Council and that of PJ by the Academy of Finland. TIMAS data were produced under support of NASA contract NAG-53032 to Lockheed Martin and NAG5-11391 to the University of Colorado. We are grateful to NASA for making TIMAS data available through CDAWeb.

The Editor in Chief thanks E. Sagaura and another referee for their help in evaluating this paper.

\section{References}

André, M. and Yau, A.: Theories and observations of ion energization and outflow in the high latitude magnetosphere, Space Sci. Rev., 80, 27-48, 1997.

Collin, H. L., Peterson, W. K., Lennartsson, O. W., and Drake, J. F.: The seasonal variation of auroral ion beams, Geophys. Res. Lett., 25, 4071-4074, 1998.

Ergun, R. E., Carlson, C. W., McFadden, J. P., et al.: FAST satellite observations of electric field structures in the auroral zone, Geophys. Res. Lett., 25, 2025-2028, 1998.

Frank, L. A. and Ackerson, K. L.: Observations of charged particle precipitation into the auroral zone, J. Geophys. Res., 76, 36123643, 1971.

Gorney, D. J., Clarke, A., Croley, D., Fennell, J., Luhmann, J., and Mizera, P.: The distribution of ion beams and conics below 8000 km, J. Geophys. Res., 86, 83-89, 1981.

Gorney, D. J., Chiu, Y. T., and Croley, Jr., D. R.: Trapping of ion conics by downward parallel electric fields, J. Geophys. Res., 90, 4205-4210, 1985.

Hallinan, T. J. and Stenbaek-Nielsen, H. C.: The connection between auroral acceleration and auroral morphology, Phys. Chem. Earth, 1-3, 26, 169-177, 2001.

Horita, R. E., Ungstrup, E., Shelley, E. G., Anderson, R. R., and Fitzenreiter, R. J.: Counterstreaming ion events in the magnetosphere, J. Geophys. Res., 92, 13 523-13 536, 1987.

Janhunen, P., Olsson, A., Mozer, F. S., and Laakso, H.: How does the U-shaped potential close above the acceleration region? A study using Polar data, Ann. Geophysicae, 17, 1276-1283, 1999.

Janhunen, P. and Olsson, A.: New model for auroral acceleration: O-shaped potential structure cooperating with waves, Ann. Geophysicae, 18, 596-607, 2000.

Janhunen, P., Olsson, A., Peterson, W. K., Laakso, H., Pickett, J. S., Pulkkinen, T. I., and Russell, C. T.: A study of inverted-V auroral acceleration mechanisms using Polar/Fast Auroral Snapshot conjunctions, J. Geophys. Res., 106, 18 995-19 011, 2001.

Janhunen, P. and Olsson, A.: A hybrid simulation model for a stable auroral arc, Ann. Geophysicae, 20, 1-14, 2002.

Janhunen, P., Olsson, A., and Laakso, H.: Altitude dependence of plasma density in the auroral zone, Ann. Geophysicae, 20, 17431750, 2002.

Janhunen, P., Olsson, A., Vaivads, A., and Peterson, W. K.: Generation of Bernstein waves by ion shell distributions in the auroral region, Ann. Geophysicae, 21, 881-891, 2003.
Johnson, R. G.: That hot ion composition, energy, and pitch angle characteristics above the auroral zone ionosphere, in: Highlatitude space plasma physics, edited by Hultqvist, B. and Hagfors, T., Plenum, New York, pp. 271-294, 1983.

Klumpar, D. M., Peterson, W. K., and Shelley, E. G.: Direct evidence for two-stage (bimodal) acceleration of ionospheric ions, J. Geophys. Res., 89, 10779-10 787., 1984.

Kondo, T., Whalen, B. A., and Yau, A. W.: Statistical analysis of upflowing ion beam and conic distributions at DE 1 altitudes, J. Geophys. Res., 95, 12 091-12 102, 1990.

Lin, C. S. and Hoffman, R. A.: Characteristics of the inverted-V event, J. Geophys. Res., 84, 1514-1525, 1979.

Lundin, R. and Eliasson, L.: Auroral energization processes, Ann. Geophysicae, 9, 202-223, 1991.

McFadden, J. P., Carlson, C. W., Ergun, R. E. et al.: Spatial structure and gradients of ion beams observed by FAST, Geophys. Res. Lett., 25, 2021-2024, 1998.

Miyake, W., Mukai, T., and Kaya, N.: On the origins of the upward shift of elevated (bimodal) ion conics in velocity space, J. Geophys. Res., 101, 26 961-26969, 1996.

Möbius, E., Tang, L., Kistler, L. M., et al.: Species dependent energies in upward directed ion beams over auroral arcs as observed with FAST TEAMS, Geophys. Res. Lett., 25, 2029-2032, 1998.

Olsson, A., Andersson, L., Eriksson, A. I., Clemmons, J., Erlandsson, R. E., Reeves, G., Hughes, T., and Murphree, J. S.: Freja studies of the current-voltage relation in substorm related events, J. Geophys. Res., 103 4285-4301, 1998.

Peterson, W. K., Collin, H. L., Doherty, M. F., and Bjorklund, C. $\mathrm{M} .: \mathrm{O}^{+}$and $\mathrm{He}^{+}$restricted and extended (bi-modal) ion conic ditributions Geophys. Res. Lett., 19, 1439-1442, 1992.

Press, W. H., Teukolsky, S. A., Vetterling, W. T., and Flannery, B. P.: Numerical Recipes in C, The art of scientific computing, 2nd ed., Cambridge, 1992.

Sagawa, E., Yau, A. W., Whalen, B. A., and Peterson, W. K.: Pitch angle distributions of low-energy ions in the near-earth magnetosphere, J. Geophys. Res., 92, 12 241-12 254, 1987.

Shelley, E. G., Simpson, D. A., Saunders, T. C., Hertzberg, E., Balsiger, H., and Ghielmetti, A. G.: The energetic ion composition spectrometer (EICS) for the Dynamics Explorer-A, Space Sci. Instrum., 5, 443, 1981.

Shelley, E. G., Ghielmetti, A. G., Balsiger, H., Black,R. K., Bowles, J. A., Bowman, R. P., Bratschi, O., Burch, J. L., Carlson, C. W., Coker, A. J., Drake, J. F., Fischer, J., Geiss, J., Johnstone, A., Kloza, D. L., Lennartsson, O. W., Magoncelli, A. L., Paschmann, G., Peterson, W. K., Rosenbauer, H., Sanders, T. C., Steinacher, M., Walton, D. M., Whalen, B. A., and Young, D. T.: The Toroidal Imaging Mass-Angle Spectrograph (TIMAS) for the Polar Mission, Space Science Rev., 71, 1-4, 1995.

Stenbaek-Nielsen, H. C., Hallinan, T. J., Osborne, D. L., Kimball, J., Chaston, C., McFadden, J., Delory, G., Temerin, M., and Carlson, C. W.: Aircraft observations conjugate to FAST: auroral arc thickness, Geophys. Res. Lett., 25, 2073-2076, 1998.

Yau, A. W., Whalen, B. A., Peterson, W. K., and Shelley, E. G.: Distribution of upflowing ionospheric ions in the high-altitude polar cap and auroral ionosphere, J. Geophys. Res., 89, 55075522, 1984.

Yau, A. W., Shelley, E. G., Peterson, W. K., and Lenchyshyn, L.: Energetic auroral and polar ion outflow at DE 1 altitudes: magnitude, composition, magnetic activity dependence, and long-term variations, J. Geophys. Res., 90, 8417-8432, 1985. 